INPLASY

PROTOCOL

To cite: Hung et al. The impact of Sofosbuvir/Velpatasvir/ Voxilaprevir treatment on serum hyperglycemia in HCV infections: A Systematic Review. Inplasy protocol 2021120109. doi:

10.37766/inplasy2021.12.0109

Received: 24 December 2021

Published: 24 December 2021

Corresponding author:

Hsuan-Yu Hung

ameeyo36@gmail.com

Author Affiliation:

School of Pharmacy, College of Pharmacy, Kaohsiung Medical University, Kaohsiung, Taiwan.

Support: No received external funding.

Review Stage at time of this submission: Data analysis.

Conflicts of interest:

None declared.

\section{The impact of Sofosbuvir/Velpatasvir/ Voxilaprevir treatment on serum hyperglycemia in HCV infections: A Systematic Review}

Hung, $\mathrm{HY}^{1}$; Chen, $\mathrm{CY}^{2}$.

Review question / Objective: To assess the possible cause of events, the incidence of grade 3 hyperglycemia after treating Sofosbuvir/Velpatasvir/Voxilaprevir in HCV infections.

Condition being studied: Sofosbuvir, velpatasvir, and voxilaprevir (SOF/VEL/VOX) is an effective, safe rescue therapy regimen for patients have previously been treated failure. Initiating Direct-Acting Antiviral (DAA) treatment for HCV infection with diabetes have experienced hypoglycemia, it could improve insulin resistance due to clean HCV. However, some studies shown that SOF/VEL/VOX has Grade 3 hyperglycemia adverse events. This finding contradicts that other DAAs studies.

Information sources: Conducting a comprehensive literature search on the pubmed, Cochrane, clinicalkey, Embase, and MEDLINE electronic databases.

INPLASY registration number: This protocol was registered with the International Platform of Registered Systematic Review and Meta-Analysis Protocols (INPLASY) on 24 December 2021 and was last updated on 24 December 2021 (registration number INPLASY2021120109).

\section{INTRODUCTION}

Review question / Objective: To assess the possible cause of events, the incidence of grade 3 hyperglycemia after treating Sofosbuvir/Velpatasvir/Voxilaprevir in HCV infections.
Condition being studied: Sofosbuvir, velpatasvir, and voxilaprevir (SOF/VEL/VOX) is an effective, safe rescue therapy regimen for patients have previously been treated failure. Initiating Direct-Acting Antiviral (DAA) treatment for HCV infection with diabetes have experienced hypoglycemia, 
it could improve insulin resistance due to clean HCV. However, some studies shown that SOF/VEL/VOX has Grade 3 hyperglycemia adverse events. This finding contradicts that other DAAs studies.

\section{METHODS}

Participant or population: Hepatitis C patients who have previously failed a direct-acting antiviral (DAA) or naive experiences.

\section{Intervention: SOF/VEL/VOX treatment}

\section{Comparator: Other DAA treatment.}

Study designs to be included: Only randomized controlled trials (RCT).

Eligibility criteria: Included articles were screened by following criteria: described the SVR12 and relapse states after SOF/ VEL/VOX treatment for HCV infection; next, the safety outcomes recorded consisted of Grade 3 level serum glucose parameters, and other grade 3 laboratory abnormalities adverse events.

Information sources: Conducting a comprehensive literature search on the pubmed, Cochrane, clinicalkey, Embase, and MEDLINE electronic databases.

Main outcome(s): Five RCTs were included in this systemic review. Overall, the safety outcomes revealed grade 3 level hyperglycemia occurred from zero to $7.27 \%$, rise to $15-25 \%$ of HCV GT3 infections, especially.

Quality assessment / Risk of bias analysis: This systematic review was performed according to the PRISMA statement guidelines and used the Cochrane Risk of Bias Tool for randomized trials (rob 2.0) to determine the quality of each included study.

Strategy of data synthesis: This integrated research data were intercepted from available articles. Describing the incidence were presented as percentages (\%), and
95\% confidence interval $(\mathrm{Cl})$ was used to present the value interval.

Subgroup analysis: This systematic review has no subgroup analysis.

Sensitivity analysis: This systematic review has no sensitivity analysis.

Country(ies) involved: Taiwan.

Keywords: Hyperglycemia; SOF/VEL/VOX; Hepatitis C; Diabetes.

Contributions of each author:

Author 1 - Hsuan-Yu Hung had contributions to the conception, design of research, the acquisition, analysis, and interpretation of data for the study.

Email: ameeyo36@gmail.com

Author 2 - Chung-Yu Chen works for revised this study critically for important intellectual content and final approval of the version to be published.

Email: jk2975525@hotmail.com 